
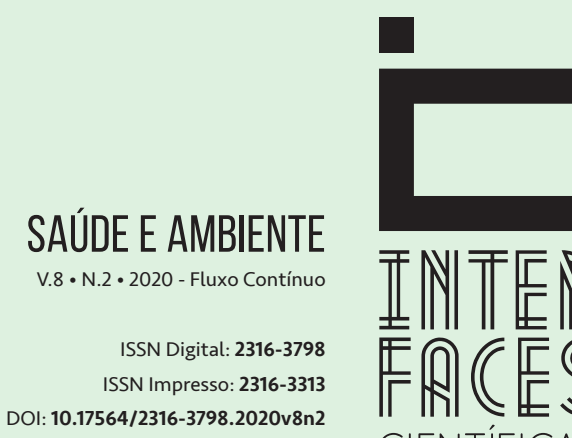

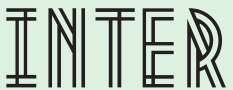

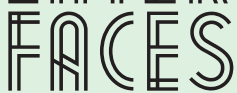

CIENTÍFICAS

\section{AVALIAÇÃO INTEGRADA DE ESCORES DE QUALIDADE DE VIDA E ESTRESSE EM PRÉ-VESTIBULANDOS}

QUALITY-OF-LIFE AND STRESS-SCORES INTEGRATED ASSESSMENT IN PRE-COLLEGE STUDENTS

EVALUACIÓN INTEGRADA DE CALIDADDE VIDA Y ESTRÉSEN ESTUDIANTES PREUNIVERSITARIOS
Marcos Antonio Almeida-Santos ${ }^{1}$ Iza Fontes Carvalho ${ }^{2}$

Marlizete Maldonado Vargas ${ }^{3}$

\section{RESUMO}

A crescente concorrência para entrada em instituições de ensino superior é um fator que pode influenciar no surgimento de estresse e consequentemente nos níveis de qualidade de vida. Embora o estado da arte existente aceite a influência simultânea desses fatores, há carência de estudos que quantifiquem essa interrelação. Este trabalho teve como objetivo elaborar uma análise combinada de escores de estressee qualidade de vida em jovens estudantes de curso preparatório para universidades, mediante a aplicação de modelos de equações estruturais. Trata-se de uma pesquisa quantitativa exploratória de corte transversal e amostra composta por 32 sujeitos. Foi utilizado o Inventário de Estresse de Lipp (ISSL) para obter os níveis de estresse e o WHOQOL-Bref para mensurar os índices de qualidade de vida. Os resultados revelaram estresse positivo em $68,75 \%$ dos sujeitos. Em relação à qualidade de vida, a maior média encontrada foi na autoavaliação $(14,13)$ e a menor foi no domínio ambiental $(11,70)$. Concluiu-se que o modelo desenvolvido de equações estruturais foi capaz de quantificar a correlação negativa entre níveis de estresse e qualidade de vida (coeficiente estandardizado $=-0.65)$ para a amostra analisada.

\section{PALAVRAS-CHAVE}

Estresse. Qualidade de Vida. Estudantes. 


\section{ABSTRACT}

This study has as scope the development of a combined analysis of stress scores and quality of life of young students preparing for entrance into universities in Brazil, by applying structural equation models. It used the Inventory of StressLipp (ISSL) to get the stress levels and the WHOQOL-Brief to measure the levels of quality of life. The results showed that stress was positive in $68.75 \%$ of respondents. Concerning quality of life, the highest average was in the self-evaluation (14.13) and the lowest average was in the environmental sphere (11.70). There was a negative correlation between stress levels and quality of life (standardized coefficient $=-0.65$ ) for the analyzed sample.

\section{KEYWORDS}

Stress. Quality of Life. Students.

\section{RESUMEN}

Este estudio tiene como escope el desarrollo de un análisis combinado de las puntuaciones de estrés y calidad de vida de los jóvenes estudiantes que se preparan para ingresar a las universidades de Brasil, mediante la aplicación de modelos de ecuaciones estructurales. Utilizó el Inventario de Estresse Lipp (ISSL) para obtener los niveles de estrés y el WHOQOL-Bref para medir los niveles de calidad de vida. Los resultados mostraron que el estrés fue positivo en el $68,75 \%$ de los sujeitos. En cuanto a la calidad de vida, el promedio más alto fue en la autoevaluación $(14,13)$ y el más bajo en la esfera ambiental $(11,70)$. Hubo una correlación negativa entre los niveles de estrés y la calidad de vida (coeficiente estandarizado $=-0.65$ ) para la muestra analizada.

\section{PALABRAS-CLAVE}

Estrés. Calidad de Vida. Estudiantes. 


\section{INTRODUÇ̧̃̃o}

O número crescente de concorrentes as vagas disponibilizadas por instituições de ensino superior (IES) exige melhor preparação dos candidatos. Essa preparação para os exames de admissão em cursos superiores faz parte do protocolo a ser cumprido pelos estudantes em busca de melhores condições de acesso ao mercado de trabalho. No período que antecede as provas, o estresse pode surgir. Os transtornos que acompanham o estresse têm sido estudados mais recentemente em adolescentes e adultos jovens (CAPANO; PACHECO, 2014; DEBNAM et al., 2016; YEAGER et al., 2016). No entanto, apesar da crescente expansão de pesquisas sobre o estresse, estudos voltados para a mensuração de sua influência durante a adolescência e a preparação para entrada no ensino superior ainda são escassos (FARIA et al., 2012).

Considera-se que a avaliação dos níveis de estresse e de qualidade de vida dos indivíduos que se preparam para ingressar em um bom curso superior são relevantes no campo da psicologia visto que o estresse é influenciado e influencia na percepção da qualidade de vida de acordo com o que aponta a literatura (AL-SMADI et al., 2017; CORREIA; LINHARES, 2014; FONSECA et al., 2014). Com os níveis de estresse altos e qualidade de vida baixos os alunos podem perder rendimento escolar e por vezes não atingir seu potencial (MOXOTÓ; MALAGRIS, 2015).

$\mathrm{O}$ estresse, inicialmente descrito como fator que origina a Síndrome de Adaptação Geral atualmente é compreendido em seu modelo quadrifásico o qual é composto pelas fases de alerta, resistência, quase-exaustão e exaustão (LIPP; GUEVARA, 1994; SELYE, 1936). As fases comportam tanto variáveis psicológicas quanto físicas que nas fases de quase-exaustão e exaustão podem aumentar os níveis de vulnerabilidade no contexto escolar (SCHERMANN et al., 2014).

A qualidade de vida (QV) por sua vez, é conceituada pela OMS como a percepção de um indivíduo em relação a seus objetivos, expectativas, padrões e preocupações no contexto cultural e dos sistemas de valores nos quais eles vivem embora exista consenso teórico em relação a seu conceito (MINAYO et al., 2000). Como o construto qualidade de vida é atribuído à percepção do indivíduo em relação a si, os estudos relacionados a QV buscam contribuir para um melhor entendimento dos processos existentes e consequentemente auxiliar no diagnóstico precoce de comprometimentos ao bem-estar, possibilitando a identificação de subgrupos vulneráveis ao desencadeamento de problemas de saúde como o estresse (SILVEIRA et al., 2013).

Pesquisas que utilizem mensurações de estresse e qualidade de vida e suas possíveis correlações, são escassas (MELO et al., 2013; RONCON et al., 2015; SANTOS; CARDOSO, 2010). Adicionalmente, esses estudos, em geral, utilizaram recursos estatísticos tradicionais, tais como teste $t$ de Student, correlação de Pearson, teste de Mann-Whitney e regressão linear. Nesse sentido, uma importante contribuição da estatística moderna, tornada acessível graças aos avanços tecnológicos em informática, foi viabilizar o emprego de modelos de equações estruturais (SEM - structural equation models). No âmbito da psicologia o uso da SEM permite o dimensionamento de construtos e a avaliação quantitativa de variáveis latentes (MONTGOMERY et al., 2010; PILATI; LAROS, 2007; REYNA et al., 2014).

No que concerne aos instrumentos validados de mensuração de estresse e de qualidade de vida utilizados no Brasil, figuram o Inventário de Sintomas de Estresse de Lipp (ISSL), referência para 
avaliar a prevalência de sintomas e o nível de estresse, e o WHOQOL-Bref, usado para avaliar a qualidade de vida dos sujeitos. Tanto o ISSL quanto o WHOQOL-Bref têm sido aplicados em diversos perfis amostrais (MOXOTÓ; MALAGRIS, 2015; PIRES et al., 2012; PONTES-FERNANDES; PETEAN, 2011).

O objetivo do presente estudo foi apresentar uma análise combinada de escores de estresse (ISSL) e qualidade de vida (WHOQOL-Bref) em jovens estudantes de curso preparatório para ingressar em instituições de ensino superior (IES), mediante a aplicação de modelo de equações estruturais.

\section{MÉTODO}

O presente estudo é quantitativo de corte transversal, com caráter exploratório cuja população foi composta por alunos de um polo de pré-vestibular público da cidade de Aracaju-SE. O critério de inclusão adotado foi cursar pré-vestibular na unidade estudada, excluindo-se da amostra indivíduos que possuíam algum comprometimento cognitivo que os impedissem de responder aos questionários. Havia 34 alunos no local e todos foram convidados a participar da pesquisa mediante leitura do termo de Consentimento Livre e Esclarecido (TCLE). Dois alunos se recusaram a participar da pesquisa, resultando em uma amostra composta por 32 indivíduos, sendo 17 do sexo masculino e 15 do sexo feminino com idade média de $22( \pm 7,629,38)$ anos.

\subsection{INSTRUMENTOS}

Foram utilizados o Inventário de Estresse de Lipp (ISSL) e o WHOQOL Bref. O ISSL visa identificar de modo objetivo a sintomatologia que o indivíduo apresenta, avaliando se este possui sintomas de estresse, qual o tipo existente (se físico ou psicológico) e a fase em que se encontra (LIPP, 2014). 0 inventário é composto por 53 itens divididos entre físicos (34) e psicológicos (19). Os sintomas são divididos em três quadros que se referem a quatro fases do estresse, onde em todos os quadros existem perguntas relacionadas a sintomas físicos e psicológicos.

0 primeiro quadro refere-se aos sintomas experimentados nas últimas 24 horas, sendo 12 sintomas físicos e três psicológicos, correspondendo à fase alerta do estresse. 0 segundo quadro indica os sintomas experimentados na última semana, ressaltando-se que nesse quadro existe uma divisão das fases do estresse em que a pessoa se encontra - porcentagens até 50 indicam que o indivíduo está na fase de resistência, caso as porcentagens ultrapassarem 50 existe indicação para a fase de quase exaustão. No último quadro, abordam-se os sintomas os sintomas vivenciados durante o último mês e correspondem à fase de exaustão.

O WHOQOL, é baseado nos pressupostos de que qualidade de vida, é um construto subjetivo, multidimensional, composto por dimensões positivas, tal como, mobilidade e negativas, como a dor. Esse instrumento foi validado por Fleck (2000), elaborado pelo grupo de Qualidade de Vida da divisão de Saúde Mental da Organização Mundial da Saúde (OMS). A versão completa do WHOQOL-100 é composta por 100 itens que avaliam quatro domínios: físico, psicológico, relações sociais e meio-am- 
biente. O WHOQOL-Bref é formado por 26 itens extraídos do WHOQOL-100 que obtiveram os melhores desempenhos psicométricos (FLECK et al., 1999, 2000).

\subsection{PROCEDIMENTOS DE COLETA DE DADOS}

Este estudo foi aprovado pelo CEP da Unit com parecer n. 45915115.7.0000.5371 e atende às normas estabelecidas pela Resolução n. 466/2012 do Conselho Nacional de Saúde para pesquisas realizadas em seres humanos. O TCLE foi assinado pelos sujeitos maiores de 18 anos e pelos responsáveis dos menores de 18, que assinaram o Termo de Assentimento antes de responder aos instrumentos.

Para a coleta de dados, realizou-se, inicialmente, uma apresentação coletiva da pesquisa na Instituição de Ensino, quando foram explicados todos os itens dos Termos de Consentimento Livre e Esclarecido e o de Assentimento (TCLE; TALE). Num segundo momento, retornou-se ao local para a aplicação dos instrumentos em blocos de até 20 sujeitos que estavam com TALE e/ou TCLE assinados. O tempo médio dispendido pelos sujeitos para responderam todas as respostas foi de 40 minutos.

\subsection{ANÁLISE DE DADOS}

As variáveis categóricas foram apresentadas em números absolutos e percentuais; e as variáveis contínuas foram apresentadas em média, desvio-padrão, mediana e intervalo inter-quartil. Para avaliar a qualidade de vida e o estresse simultaneamente, optou-se por modelos de equações estruturais ou SEM (HOOPER et al., 2008), onde se podem incluir essas variáveis como fatores - ou dimensões - latentes. Diversos modelos foram elaborados e analisados quanto à sua adequação metodológica.

Por intermédio do SEM, obteve-se a multiplicidade de dados, mesmo tratando-se de uma amostra pequena. Foi possível estimar o grau de adequação de um modelo complexo, além de viabilizar uma análise exploratória de fatores incluídos em cada modelo e dimensionar o efeito desses fatores sobre variáveis latentes, isto é, que não foram diretamente mensuradas, porém colimam subjetivamente determinados agregados de fatores.

As variáveis, atenção sintoma físico e psicológico (afp), resistência sintoma físico e psicológico (rfp) e exaustão (efp), que variaram entre zero e 14, representaram o nível global de estresse, e as variáveis auto avaliação, dimensão física, psíquica, ambiental e social, em escala de zero a 20 (tendo variado entre seis e 20), representaram o nível global de qualidade de vida. Conforme estudos contendo modelos e instrumentos semelhantes esperava-se uma correlação negativa entre estresse e qualidade de vida (FOOLADI et al., 2014; LIU et al., 2015).

As estimativas foram realizadas pelo método de máxima verossimilhança ("maximum likelihood estimation"), considerado o mais apropriado em virtude de sua robustez diante de potencial violação do pressuposto de normalidade de distribuição dos parâmetros. Em resumo, o modelo criado resultou em duas variáveis latentes e oito variáveis endógenas: a dimensão da variável latente "estresse" encampou três variáveis endógenas e a dimensão da variável latente "qualidade de vida" encampou cinco variáveis endógenas. Foram considerados de significância estatística os valores de $p<0,05$, assim como intervalos de confiança a 95\% sem encampar o número zero, havendo completa correspondência entre ambos. 
Os resultados foram apresentados graficamente, incluindo os coeficientes estandardizados e respectivos valores de $\mathrm{p}$. A estandardização dos coeficientes apresenta entre as vantagens a homogeneização de variância das variáveis latentes, a estabilidade dos valores (pois independem da categoria utilizada como referência) e a possibilidade de comparar coeficientes oriundos de diferentes escalas.

Algumas variáveis tiveram códigos invertidos, a fim de representar a variável latente na direção adequada. Isso ocorreu para três questões: três, quatro e 26 do instrumento WHOQOL-Bref. Em decorrência da potencial associação entre estresse e qualidade de vida, buscou-se desenvolver um modelo que contemplasse a covariância entre ambas as variáveis latentes. 0 erro padrão foi estimado pela matriz de informação observada. Utilizou-se o programa estatístico Stata versão 14,1.

\section{RESULTADOS E DISCUSSÃO}

Todos os alunos pertenciam à rede estadual de ensino, em sua maioria residiam em Aracaju (70,97\%), não exerciam atividade remunerada (68,75\%), utilizavam transporte público (68,75\%), não possuíam filhos (87,50\%), não praticavam atividade física $(59,38 \%)$, não fumavam $(96,88 \%)$ e não faziam uso de bebidas alcoólicas (78,13\%).

A mensuração do estresse revelou-se positiva em $68,75 \%$ dos entrevistados. A fase de resistência (56,35\%) e os sintomas psicológicos $(46,88 \%)$ foram os componentes predominantes. Os principais sintomas psicológicos foram: pensar constantemente em um só assunto ( $71,88 \%)$; ter dúvida quanto a si próprio (59,38\%); fugir de tudo (56, 25\%); angústia/ ansiedade diária e vontade súbita de iniciar novos projetos. Em relação à qualidade de vida, a maior média encontrada na escala de 20 foi na auto avaliação $(14,13)$ e a menor foi o domínio ambiental $(11,70)$.

Em estudo longitudinal de um ano realizado por Calais Montanhaur e Salgado (2018) com 71 estudantes do terceiro ano do ensino médio em escolas de ensino público e privado os resultados convergiram com os obtidos no presente artigo visto que foi possível observar a predominância dos sintomas psicológicos na fase de resistência o que pode ser ocasionado por desgaste ante agentes estressores.

Os valores médios dos escores de estresse e qualidade de vida, assim como o desvio-padrão e percentis, estão apresentados na Tabela 1.

Tabela 1 - Padrões de distribuição dos escores de estresse e qualidade de vida

\begin{tabular}{ccccccccc}
\hline & afp & rfp & efp & Auto avaliação & Físico & Psíquico & Ambiente & Social \\
\hline Média & 4,53 & 5,22 & 5,59 & 14,13 & 13,23 & 12,72 & 11,71 & 13,96 \\
Desvio-padrão & 2,57 & 2,73 & 3,46 & 2,67 & 2,89 & 3,19 & 2,30 & 3,19 \\
Mediana & 5,00 & 5,50 & 6,00 & 14,00 & 13,43 & 12,67 & 12,00 & 13,33 \\
Percentil 25 & 2,00 & 3,00 & 2,50 & 12,00 & 11,43 & 10,67 & 9,50 & 12,00 \\
\hline
\end{tabular}




\begin{tabular}{ccccccccc}
\hline & afp & rfp & efp & Auto avaliação & Físico & Psíquico & Ambiente & Social \\
\hline Percentil 75 & 6,00 & 7,00 & 8,00 & 16,00 & 15,43 & 15,33 & 13,32 & 16,00
\end{tabular}

* afp = atenção, sintoma físico e psicológico; $r f p$ = resistência, sintoma físico e psicológico; efp = exaustão, sintoma físico e psicológico.

Fonte: Dados da pesquisa.

Os escores obtidos para o estresse na fase alerta, resistência e exaustão, englobando fatores físicos e psicológicos variaram entre 0 e 14. Para a variável qualidade de vida, encampando autoavaliação, físico, psíquico, social e ambiente, variaram entre 15,5 e 20. Uma análise visual comparativa do padrão de distribuição dos escores de estresse e de qualidade de vida é apresentada mediante “boxplots" (FIGURA1).

Figura 1 - Escores de estresse e qualidade de vida

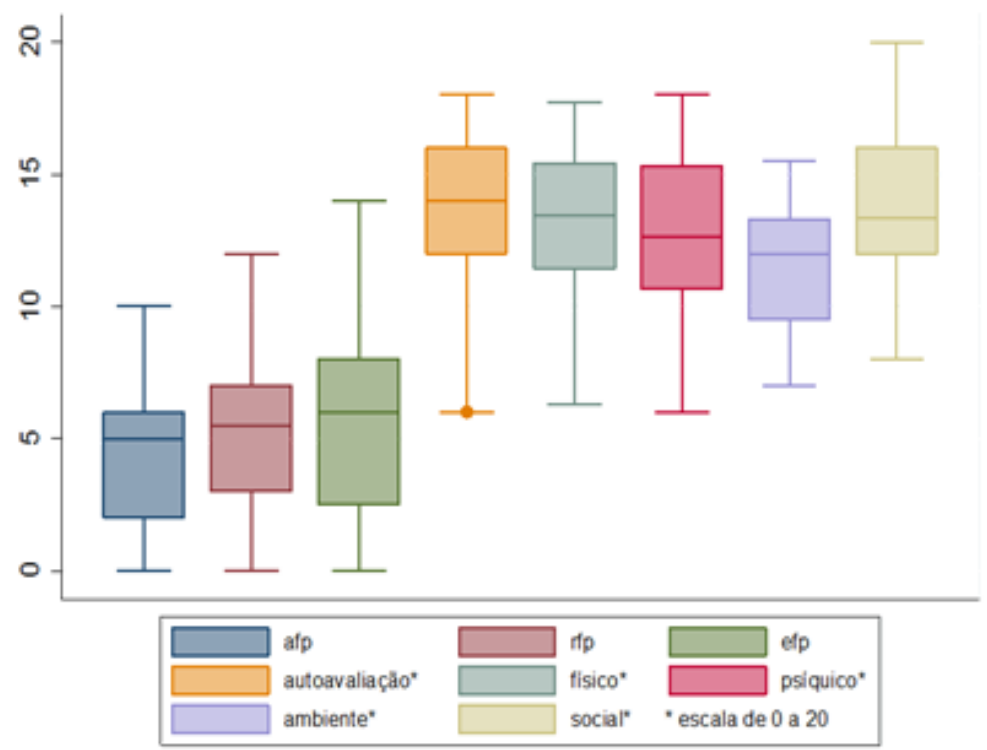

*afp = fase de alerta, sintoma físico e psicológico; rfp = fase de resistência, sintoma físico e psicológico; efp = fase de exaustão, sintoma físico e psicológico.

Fonte: Dados da Pesquisa

Um modelo parcimonioso e de fácil convergência foi criado (FIGURA 2). Novos ajustes de adequação foram utilizados, mas não apresentaram parâmetros indicativos de melhoria do modelo. 0 detalhamento dos coeficientes, incluindo o erro padrão obtido pela matriz de informação observada (OIM), assim como valores de p e respectivos intervalos de confiança a 9\% são apresentados na Tabela 2. 
Tabela 2 - Coeficientes estandardizados para estresse e qualidade de vida

\begin{tabular}{|c|c|c|c|c|c|}
\hline & $\begin{array}{l}\text { Coeficiente } \\
\text { estandardizado }\end{array}$ & Erro padrão & \multicolumn{2}{|c|}{$\begin{array}{c}\text { Intervalo de confiança } \\
95 \%\end{array}$} & Valor de $\mathrm{p}$ \\
\hline \multicolumn{6}{|l|}{ Estresse } \\
\hline afp & 0,47 & 0,16 & 0,16 & 0,78 & $p=0,003$ \\
\hline $\mathrm{rfp}$ & 0,77 & 0,14 & 0,48 & 1,05 & $p<0,001$ \\
\hline efp & 0,95 & 0,12 & 0,71 & 1,19 & $p<0,001$ \\
\hline \multicolumn{6}{|l|}{ Qualidade de vida } \\
\hline Auto avaliação & 0,81 & 0,7 & 0,67 & 0,95 & $p<0,001$ \\
\hline Físico & 0,94 & 0,38 & 0,87 & 1,02 & $p<0,001$ \\
\hline Psíquico & 0,87 & 0,06 & 0,76 & 0,98 & $p<0,001$ \\
\hline Social & 0,66 & 0,11 & 0,44 & 0,87 & $p<0,001$ \\
\hline Ambiente & 0,75 & 0,09 & 0,57 & 0,93 & $p<0,001$ \\
\hline
\end{tabular}

* afp = alerta, sintoma físico e psicológico; rfp = resistência, sintoma físico e psicológico; efp = exaustão, sintoma físico e psicológico, autoav = autoavaliação.

Fonte: Dados da Pesquisa

Em relação aos testes de adequação modelo, obteve-se CFI (índice de ajuste comparativo) igual a 0,85, TLI (Índice de Tucker-Lewis) igual a 0,78 e o coeficiente de determinação igual a 0,992, indicando que quase a totalidade da variação dos fatores é explicada pelo próprio modelo. Estes valores são discretamente inferiores aos parâmetros ideais de adequação do modelo. Porém, há que considerar-se que índices ideais são dificilmente encontrados em amostras de pequeno tamanho e a consistência do racional teórico tem sido mencionada como um fator de maior relevância (HOOPER et al., 2008), a ponto de a necessidade de aplicação sistemática desses testes ser discutível (BARRETT, 2007).

É importante ressaltar que a correlação negativa (-0.65) entre estresse e qualidade de vida não representa forçosamente um nexo causal. Também, por tratar-se de estudo transversal, estabelecer relações de temporalidade entre as variáveis latentes "qualidade de vida" e "estresse" estariam fora do escopo da presente pesquisa. 0 estudo transversal tem como principais vantagens o alto potencial descritivo o qual subsidia o planejamento dos programas e ações, bem como a simplicidade analítica e a possibilidade para demonstrar as características da população e em subgrupos.

Foi possível metodologicamente quantificar associação entre as variáveis e ao mesmo tempo identificar sua direção. Assim, para cada incremento de uma unidade padronizada na qualidade de vida, ocorre uma redução de zero vírgula sessenta e cinco no estresse. Por sua vez, para cada redução de uma unidade padronizada no estresse, ocorre incremento de zero vírgula sessenta e cinco no estresse na qualidade de vida. De igual modo, ao compararmos os coeficientes padronizados, tornou-se possível verificar que essa correlação, além de significante sob o ponto de vista da estatística, é de 
considerável magnitude, sendo inclusive superior àquela entre afp e estresse, e de semelhante expressão se comparada com a relação entre a dimensão social e qualidade de vida (FIGURA 2).

Figura 2 - Modelo de equações estruturais para análise combinada entre escores de qualidade de vida e de estresse

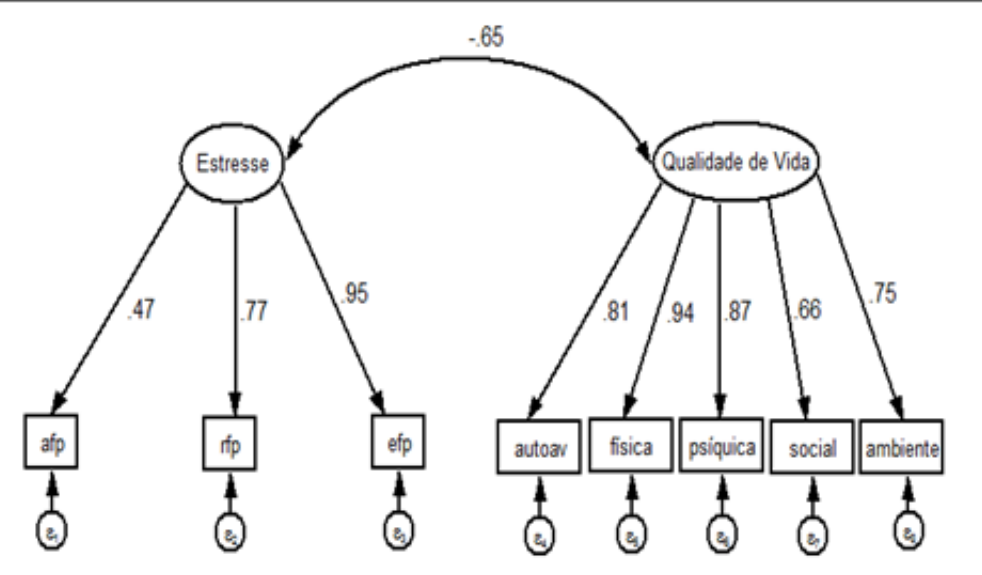

*Os valores representam coeficientes estandardizados, permitindo comparações entre diferentes escalas. Fonte: Dados da Pesquisa

Novos modelos foram testados na presente pesquisa. A avaliação do efeito de uma potencial modificação do modelo, como, por exemplo, a inclusão de relação de variância-covariância entre múltiplas variáveis exógenas, não resultou em convergência de estimativas, algo que indica não se tratar de modelo mais apropriado. A mensuração dos índices de modificação sugeriu uma forte relação de interdependência entre as fases de resistência e exaustão física e psicológica (rfp e efp).

Entretanto, novos ajustes de adequação do modelo, quando se inseriu esse aspecto, também não apresentaram parâmetros indicativos de melhoria do modelo. Igualmente, o critério de informação de Akaike não apresentou redução adicional. Em consequência, consideramos o presente modelo como o de melhor adequação e o mais parcimonioso.

Uma potencial limitação do presente estudo é o reduzido tamanho amostral, que impediu a análise de subgrupos.

\section{CONCLUSÃO}

O modelo desenvolvido foi capaz de quantificar, com satisfatória robustez, a influência do estresse, o nível de qualidade de vida e a interação entre ambos fatores em estudantes de pré-vestibular. 
Houve uma correlação negativa entre níveis de estresse e qualidade de vida, avaliados mediante a construção de variáveis latentes.

A obtenção de coeficientes padronizados mediante modelos de equações estruturadas permite utilizar essa metodologia em situações complexas, onde são utilizados múltiplos questionários em diferentes escalas.

\section{REFERÊNCIAS}

AL-SMADI, A. M. et al. Anxiety, estresse, and quality of life among Iraqi refugees in Jordan: A cross sectional survey. Nurs. Health Sci., v. 19, n. 1, p. 100-104, 2017.

BARRETT, P. Structural equation modelling: Adjudging model fit. Pers. Indiv. Differ., v. 42, n. 5, p. 815-824, 2007.

CALAIS, S. et al. Stress e qualidade de vida de pré-vestibulandos: Estudo longitudinal. Rev. Bras. Terap. Comp. Cogn., v. 19, n. 4, p. 62-73, 2018.

CAPANO, A.; PACHECO, A. Estrés y violencia domestica: um estudio en adultos referentes de niños, niñas y adolescentes. Cienc. Psicol., v. 8, n. 1, p. 31-34, 2014.

CORREIA, L. L.; LINHARES, M. B. M. Enxaqueca e estresse em mulheres no contexto da Atenção Primária. Psicol. Teoria Pesq., v. 30, n. 2, p. 145-152, 2014.

DEBNAM, K. et al. The role of stress and spirituality in adolescent substance use. Subst. Use Misuse, v. 51, n. 6, p. 733-741, 2016.

FARIA, R. R. et al. O estresse entre vestibulandos e suas relações com a família e a escolha profissional. Psicol. Argum., v. 30, n. 68, p. 43-52, 2012.

FLECK, M. P. A. et al. Aplicação da versão em português do instrumento abreviado de avaliação da qualidade de vida” WHOQOL-bref”. Rev. Saúde Públ., v. 34, n. 2, p. 178-183, 2000.

FLECK, M. P. A. et al. Desenvolvimento da versão em português do instrumento de avaliação de qualidade de vida da OMS (WHOQOL-100). Rev. Bras. Psiquiatr., v. 21, n. 1, p. 19-28, 1999.

FONSECA, M. et al. Avaliação da Qualidade de Vida Infantil: 0 Inventário Sistémico de Qualidade de Vida para Crianças. Psicol. Refl. Crítica, v. 27, n. 2, p. 282-290, 2014. 
FOOLADI, N. et al. Comparison of depression, anxiety, stress and quality of life in drug abusers with normal subjects. Procd. Soc. Behv., v. 159, n. 1, p. 712-717, 2014.

HOOPER, D. et al. Structural Equation Modelling : Guidelines for determining model fit structural equation modelling : guidelines for determining model fit. Electron. J. Bus. Res., v. 6, n. 1, p. 53-60, 2008.

LIPP, M. E. N. Inventário de Sintomas de Estresse para Adultos de Lipp- Manual. São Paulo-SP: Casa do Psicólogo, 2014.

LIPP, M. E. N.; GUEVARA, A. J. H. Validação empírica do inventário de sintomas de stress. Brazil. Est. Psicol., v. 1, n.3, p. 43-49, 1994.

LIU, C. et al. Factors related to health-related quality of life among Chinese psychiatrists: occupational estresse and psychological capital. BMC Health Serv. Res., v. 15, n. 1, p. 20, 2015.

MELO, R. L. P. et al. O Efeito do Eestressee na Qualidade de Vida de Idosos: O Papel Moderador do Sentido de Vida. Psicol. Refl. Crítica, v. 26, n. 2, p. 222-230, 2012.

MINAYO, M. C. S. et al. Qualidade de vida e saúde: um debate necessário. Ciênc. saúde col., v. 5, n. 1, p. 7-18, 2000.

MONTGOMERY, C. et al. Le stress, les stratégies d'adaptation, le locus de contrôle et l'épuisement professionnel chez les professeurs universitaires francophones. Can. J. High. Educ., v. 40, n. 1, p. 69-99, 2010.

MOXOTÓ, G. F. A.; MALAGRIS, L. E. N. Avaliação de treino de controle do estresse para mães de crianças com transtornos do espectro autista. Psicol. Refl. Crítica, v. 28, n. 4, p. 772-779, 2015. PILATI, R.; LAROS, J.A. Modelos de equações estruturais em psicologia: conceitos e aplicações.

Psicol. Teoria Pesq., v. 23, n. 2, p. 205-216, 2007.

PIRES, L. et al. Qualidade de vida de adolescentes modelos profissionais. Psicol. Teoria Pesq., v. 28, n. 1, p. 71-76, 2012.

PONTES-FERNANDES, A. C.; PETEAN E. B. L. Sobrecarga emocional e qualidade de vida em mães de crianças com erros inatos do metabolismo. Psicol. Teoria Pesq., v. 27, n. 4, p. 459-65, 2011.

REYNA, C. et al. Exploratory structural equation modeling of the general decision-making style inventory. Rev. Psicol., v. 23, n. 1, p. 33-39, 2014. 
RONCON, J. et al. Qualidade de vida, morbilidade psicológica e estresse familiar em idosos residentes na comunidade. Psicol. Teoria Pesq., v. 31, n. 1, p. 87-96, 2015.

SANTOS, A. F. D. O.; CARDOSO, C. L. Profissionais de saúde mental: estresse, enfrentamento e qualidade de vida. Psicol. Teoria Pesq., v. 26, n. 3, p. 543-548, 2010.

SCHERMANN, L. B. et al. Estresse em adolescentes: estudo com escolares de uma cidade do sul do Brasil. Brazil. Aletheia, n. 43-44, p. 160-173, 2014.

SELYE, H. A syndrome produced by diverse nocuous agents. Nature, v. 138, n. 3479, p. 32, 1936.

SILVEIRA, M. F. et al. Qualidade de vida entre adolescentes: estudo seccional empregando o SF-12. Ciênc. Saúde Col., v. 18, n. 7, p. 2007-2015, 2013.

YEAGER, D. S. et al. How to improve adolescent stress responses: insights from integrating implicit theories of personality and biopsychosocial models. Psychol. Sci., v. 27, n. 8, p. 1078-1991, 2016. 
1 Doutor em Ciências da Saúde; Médico; Professor do Programa de Pós graduação em Saúde e Ambiente, Universidade Tiradentes - UNIT/SE.

E-mail: marcosalmeida2010@yahoo.com.br

2 Mestre em Saúde e Ambiente; Psicóloga. E-mail: carvalho.ifontes@gmail.com

3 Doutora em Psicologia Ciência e Profissão; Psicóloga; Professora do Programa de Pós graduação em Saúde e Ambiente, Universidade Tiradentes - UNIT/SE.

E-mail: marlizete@uol.com.br

\section{(ㅇ) (1) (-)}

Este artigo é licenciado na modalidade acesso abertosob a Atribuição-Compartilhalgual CC BY-SA

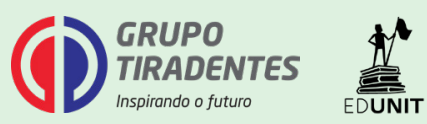

